CONTROL OF MACHINE FUNCTIONS OR TRANSPORT SYSTEMS*

M. D. Woodley, M. J. Lee, J. Jäger, ' A. S. King

\title{
Stanford Linear Accelerator Center
} P.O. Box 4349

Stanford University

Stanford, CA 94305

A computer code, COMFORT, has been developed at SLAC for on-line calculation of the strengths of magrietic elements in an electron storage ring or transport beam line, subject to first order fitting constraints on the ring or beam line parameters. This code can also be used off-line as an interactive lattice or beam line design tool.

\section{Intraduction}

COMFORT (control of Machine Functions, arbits and Trajectories) is a computer modeling code for electron storage rings. linacs and beam transport sustems which has been developed to be integrated into the control systems for PEP, SPEAR and SLC.

As part of a control system', comfORT can be used on-line to compute the magnetic element strengths needed to achieve desired first order beam and lattice parameter values such as tunes, beta and eta functions and beam sigma and transfer matrix elements. It can also be used to compute dipole corrector strengths which minimize orbit distortians and dispersion in storage rings, and to steer beams in insertions and transport systems.

Like TRANSPORT ${ }^{2}$ and MAGIC ${ }^{3}$, COMFORT can also be used off-line for the design and study of storage $r$ ing lattices and transport beam lines. We are currently working on incorporating SLAC's Unified Graphics System ${ }^{4}$ into COMFORT to create a truly interactive beam line and lattice design tool.

The advantages of COMforT are compactness and modularity, making this program easy to maintain and modify. In addition. complete documentation within the source code itself helps to make the program portable and easily adaptable to both stand-alone and subprocess applications. The purpose of this paper is to describe both the physical and mathematical structure of COMFORT, and to describe how COMFORT has been incorporated into the control program for the SLC Bamping Rings.

\section{Physical structure}

COMFORT was written originally to run on a DEC POP-11/780 ("VAX") minicomputer using the FORTRAN language: in a "stand-alone" configuration the source code is approximately 5700 lines long. Although FORTRAN-77 was available, whenever possible FORTRAN-66 (ANSI 1965 standard) has been used in order to insure partability of the program to other systems. The only exception is the use of FORTRAN-77 "PARAMETER" statements which make COMFORT a variable size program. Arrays containing data for each element type (bend, quad, etc.) have dimension declarators which are integer variables whose values are established at compile-time via an included tile of PARAMETER statements. Hence the user can define how many of each type of element will be allowed in his version of COMFORT by simply editing the PARAMETER statement file

i CERN, Geneva, Switzerland.

* Work supported by the Department of Energy, Contract DE-ACO3-765F00515. and recompiling. Double precision variables and function calls have been used throughout the program to minimize round-aff and truncation errors in the computations. Our stand-alone version of COMFORT, when compiled and linked, loads into approximately 85 Kilobytes of storage; the sub-process version of COMFORT which has been incorporated into the control program for the SLC Damping Rings has been reduced to the minimum size needed to model these rings and loads into less than 78 kilobytes.

Another natable feature of COMFORT is its error handling capability. Over fifty different lypes of error, ranging from errors in the input dataset to errors encountered during computation can be detected. When an error condition is encountered COMFORT flags the error by type, issues an informative message and returns control to the main program via a series of alternate returns. This feature enables COMFORT to act as a subprocess within the control system -- the error flag can canveniently be passed back to the parent control program for appropriate action.

Internally, comfort is divided into four subsections, each having a distinct function as described briefly below:

Input/output. COMFORT is a data set driven program: element definitions. beam line arrangement, fitting constraints and computation control flags are all contained in an input dataset in a fixed format which is similar to that of MAGIC. Most of the commands available in MAGIC have been incorporated into COMFORT, with two major improvements: combined function magnets and accelerator sections have been added. The Input subsection of COMFORT handles the reading of this dataset and the loading of all data arrays. Output is controlled from a single routine so that all output formatting statements are centrally located for easy access and modification.

Fitting. Fitting in COMFORT is accomplished by adjusting the values of the quantities which have been declared as variables to minimize the following function:

$$
\sum_{i=1}^{N} W_{i}\left(G_{i}-F_{i}\right)^{2}
$$

where

$N \equiv$ number of requested first order constraints

$W_{i} \equiv$ specified weighting factor for $i$ th constraint

$G_{i}$ Ispecified goal value for $i^{\text {th }}$ constrained quantity

$F_{i} \equiv$ present value of $i^{\text {th }}$ constrained quantity

The actual minimization is done using the variable Metric Method for Minimizations (VMM), with the required partial derivatives of constrained quantities with respect to the variables computed numerically. We have found this procedure to be satisfactory for all practical applications and it makes the addition of new functions to be fitted simple. 
Computation of Twiss Parameters. COMFORT computes the Twiss parameters ( $B, n, a, \pi^{\prime}$, tune, etc.) of the specified beam line in a single subrautine. The output of this routine is an array of Twiss parameter values evaluated at the beginning of the beam line and at the exit of each element. We will discuss how these computations are made in the "Mathematical structure" section of this paper.

Further Computations Using Twiss Parameters. depending on which computation control flags have been set in the input dataset. COMFORT will use the Twiss parameter array to compute storage ring parameters, closed orbit and dispersion correction matrices ${ }^{6}$, natural chromaticities ${ }^{7}$. synchrotron integrals ${ }^{8}$ and partition functions, and trajectory correction matrices for the system under study. The ready accessibility of the Twiss parameter array in COMfORT's main program simplifies the addition of customized computations. An addition can be made by uriting a subroutine to perform the desired computations, calling it from the main program, and passing the Twiss parameter array to it.

\section{Mathematica! structure}

The prime function of COMFORT is the computation of the Twiss parameters of a given system; discussion of subsequent computations which are performed by CampORT using these parameters is best left to the references cited above.

For machines with zero coupling between the horizontal and vertical planes cOMFORT considers seperately the transfer of particle coordinates $\left(x, x^{\prime}, d E / E\right)$ and $\left(y, y^{\prime}, d E / E\right)$ through the system, where $E$ is the energy of the central trajectory and $d E$ is the particle energy deviation. In the absence of energy gain the beam sigma matrix is given by:

$$
\left[\begin{array}{ll}
\sigma_{11} & \sigma_{12} \\
\sigma_{21} & \sigma_{22}
\end{array}\right]=\left[\begin{array}{cc}
\epsilon B & -\epsilon \alpha \\
-\epsilon \alpha & \epsilon \gamma
\end{array}\right]
$$

where $f$ is the beam emittance. Substitution of Eq. (2) into the well known relationship

$$
\sigma_{s}=R \sigma_{0} R^{T}
$$

yields the transfer equation:

$$
\left[\begin{array}{cc}
\beta & -\alpha \\
-\alpha & \gamma
\end{array}\right]_{S}=R\left(\begin{array}{cc}
\beta & -a \\
-\alpha & \gamma
\end{array}\right]_{0} R^{\top}
$$

where $R$ is the transfer matrix from 0 to $s$. Since $\epsilon$ scales with beam energy as $1 / E$, we define a new transfer matrix M where:

$$
M_{i j}=\left(E_{5} / E_{0}\right) / Z^{2} R_{i j} \text {. }
$$

Replacing $R$ with $M$ in Eq. (3) and rearranging terms yields a transfer equation which is valid in the presence of energy gain:

$$
\left[\begin{array}{l}
B \\
a \\
\gamma
\end{array}\right]_{S}=S\left[\begin{array}{l}
B \\
\alpha \\
\gamma
\end{array}\right]_{0}
$$

where

$$
S \equiv\left(\begin{array}{ccc}
M_{11} 2 & -2 M_{11} M_{12} & M_{12}{ }^{2} \\
-M_{2}, M_{1}, & M_{1}, M_{22}+M_{12} M_{2}, & -M_{12} M_{22} \\
M_{21}{ }^{2} & -2 M_{22} M_{21} & M_{22}{ }^{2}
\end{array}\right] .
$$

The betatron tune shift $\Delta \nu$ is given by:

$$
2 \pi \Delta \nu=\tan ^{-1}\left[M_{12} /\left(\beta_{0} M_{11}-\alpha_{0} M_{12}\right)\right] \text {. }
$$

COMFORT allows three distinct types of beam line: insertion, cell and half cell. The only difference in the way COMFORT treats the different types is how the values of the iwiss parameters at the beginning of the beam line are determined.

The insertion stype of beam line is used to model linac sections and beam transport systems; the user must provide the Twiss parameter values at the beginning of the beam line in the input dataset.

The cell type of beam line is used to model storage ring lattices which consist of one or more full superperiods. Once the system transfer matrix $S$ (Eq. (5)) for a full superperiod has been computed, COMFORT finds the values of the Twiss parameters (satisfying appropriate boundary conditions) at the beginning of the superperiod:

$$
\left.\begin{array}{l}
B_{0}=\left|S_{12}\right|\left[1-\left(\left(S_{11}+S_{22}\right) / 2\right)^{2}\right] 1 / 2 \\
a_{0}=\beta_{0}\left(S_{11}-S_{22}\right) /\left(2 S_{12}\right) \\
n_{0}=\left(S_{13}\left(1-S_{22}\right)+S_{23} S_{12}\right) /\left(2-S_{11}-S_{22}\right) \\
n_{0^{\circ}}=\left(S_{23}\left(1-S_{11}\right)+S_{13} S_{21}\right) /\left(2-S_{11}-S_{22}\right)
\end{array}\right\}
$$

The half cell type of beam line is used to model storage ring lattices which consist of one or more superperiods having mid-point symmetry. The user enters the lattice for half of a superperiod in the input dataset; COMFORT then computes $S$ for a full superperiod by reflecting the half superperiod lattice about its end-point:

$$
s=\bar{c} c
$$

where $\bar{C}$ is the reflection of $\mathcal{C}$ given by

$$
\bar{c}=\left(\begin{array}{ccc}
c_{22} & c_{12} & c_{12} c_{23}-c_{22} c_{13} \\
c_{21} & c_{11} & c_{11} c_{23}-c_{21} c_{13} \\
0 & 0 & 1
\end{array}\right] .
$$

once the system transfer matrix $S$ has been computed, Eq. (7) is used to find the Iwiss parameters at the beginning of the lattice.

With the values of the rwiss parameters at the beginning of the beam line established, COMFORT uses Eqs. (4) and (5) and the appropriate single-element transfer matrices to step through the beam line, computing the Twiss parameters at the exit of each element as well as at the fit points where the values of the constrained quantities $F_{i}$ are to be evaluated.

\section{COMFORI IMPLEMENTATION}

COMFORT has been incorporated into the control system for the SLC Damping Rings to find the strengths of six quadrupole families which will yield desired ring parameter values (betatron tunes, eta functions at injection and extraction points, etc.) which have been entered by the operator from a touch panel, and to do closed orbit and dispersion correction. COMFORT has been interfaced to the parent control structure via a oriver Routine that handles touch panel $1 / 0$. The simplest way to describe the integration of COMFORT into the control program is by describing the actual contral process as shown in Fig. 1 . 


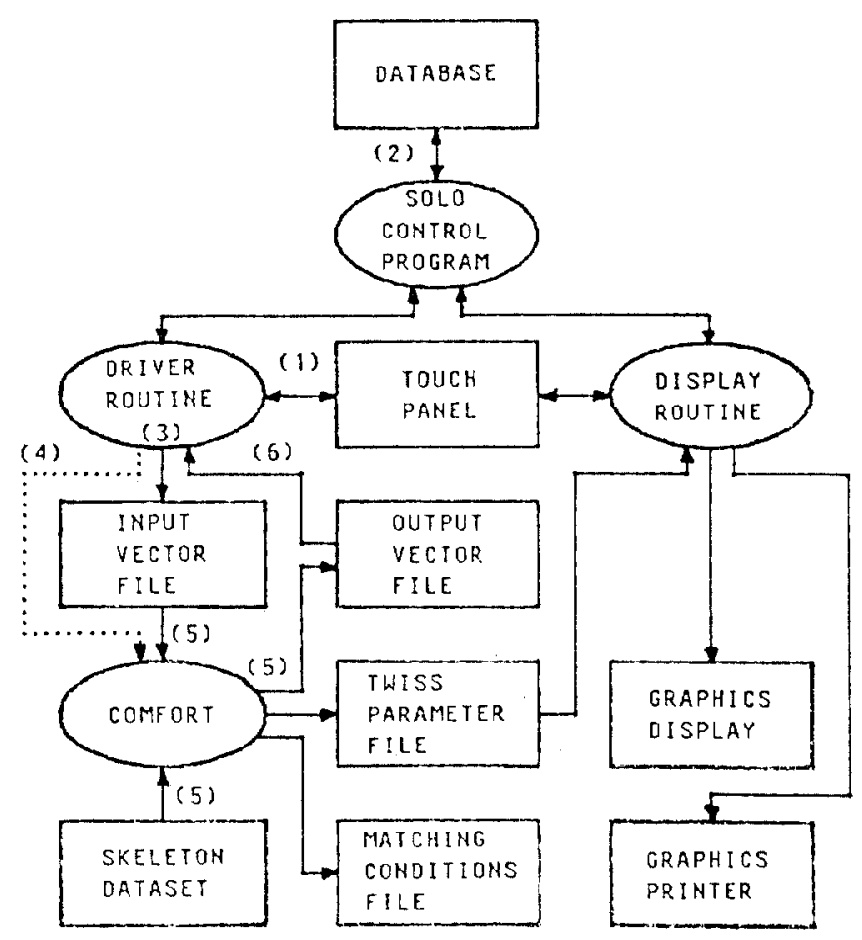

Fig. 1: SLC Damping Rings control system block diagram.

Step 1 The operator enters the desired ring parameters and/or requests for orbit and dispersion correction via a touch panel.

Step 2 The oriver Routine, using utility programs which reside in the Solo Control Program (SCP), queries the database for the present strengths of the six quadrupole lamilies, the injection energy, position monitor readings and status, corrector settings and status and other necessary system parameters.

Step 3 The Oriver Routine loads the information gathered in step 2 into a special file called an input vector file for comfORT to pick up.

Step 4 Tlae Oriver Routine initiates the COMFORT subprocess. This is shown in Fig. I as a datted line to 1 liustrate that there is no direct communication between these routines; the oriver Routine simply schedules COMFORT for execution.
Step 5 COMFORT reads a "skeleton" input dataset which contains the non-adjustable parameters of the Damping Rings (magnet lengths, etc.) and the present values of all adjustable quantities (quadrupole strengths, etc.). Next, COMFORT reads the input vector file and replaces the values read into memory from the skeleton dataset with their corresponding input vector file values. COMFORT then computes the strengths of quadrupoles and/or correctors which yield the lattice and/or orbit parameters specified in the input vector file. When the solution is found, COMFORT writes the results into three files: an output vector file containing the strengths computed for the six quadrupole families and/or corrector strengths; a file containing the Tuiss parameters evaluated at the injection and extraction points to the Rings to be used for matching to the injection and extraction beam lines, and, a file containing the Twiss parameters for the $R$ ings and any other parameter values requested (storage ring parameters, synchrotron integrals, etc.).

Step 6 The Driver Routine reads the output vector file and loads the values into the database via the SCP routines.

Displays for the Damping Rings are handled by a Display Routine which reads the Twiss parameter file created by COMFORT and formats the data for output to either a graphics display device or a graphics printer.

\section{References}

1. J. C. Sheppard, R. H. Helm, M. J. Lee and $M$. D. Woodley, "On-line Control Models for the Stanford Linear collider", to be published in the Proceedings of this conference.

2. K. L. Brown, F, Rothacker, D. C. Carey and Ch. Iselin, "TRANSPORT, A computer Program for Designing Charged Particle Beam Transport Systems", SLAC-91, Rev. 2, May 1977.

3. A. S. King, M. J. Lee and $W$. W. Lee, "MAGIC, A Computer Code for Design Studies of Insertions and Storage Rings", SLAC-183, August 1975.

4. R. C. Beech, "The SLAC Unified Graphics system, Ver. 2", SLAC-CGTM-170, Feb. 1976.

5. W. C. Davidon, "Variable Metric Method for Minimization", All-5990, Rer. 2, Feb. 1966.

6. A. W. Chao, M. J. Lee, E. Linstadt and $N$. Spencer, "On-line Orbit and Dispersion Correction Schemes for PEP", PEP-Note-318, OCt. 1979.

7. W. Hardt, J. Jäger and 0 . Möhl, "A General Analytical Expression for the Chromaticity of Accelerator Rings", PS/LEA/Note 82-5, Nov. 1982.

8. R. H. Helm, M. J. Lee, P. L. Morton and M. Sands, "Evaluation of Synchrotron Radiation Integrals", Proceedings of 1973 Particle Accelerator Conference, San Francisco. 